M.A. Khan

J. Singh

B. Dhillon

Princess Alexandra Eye

Pavilion

Edinburgh EH3 9HA, UK

M.A. Khan, DO, FRCSEd Princess Alexandra Eye Pavilion

Chalmers Street

Edinburgh EH3 9HA, UK

Tel: +44(0)1315361674

Fax: +44(0)1315361574

Received: 7 April 1999

Accepted in revised form: 30 September 1999

\section{Rifabutin-induced uveitis with inflammatory vitreous infiltrate}

\author{
Abstract \\ Purpose To describe an ocular complication of \\ rifabutin therapy in the treatment of \\ Mycobacterium avium complex (MAC) \\ infection in the absence of HIV infection or \\ AIDS. \\ Methods Three patients on rifabutin therapy \\ for MAC chest infection developed anterior \\ uveitis with vitreous infiltrates. The clinical \\ course and visual outcome are discussed. \\ Results The ocular signs of anterior segment \\ inflammation along with vitreous infiltrate \\ resolved on topical steroid therapy without \\ long-term visual sequelae. \\ Conclusion This report highlights the potential \\ of uveitis in patients treated for MAC \\ infection with rifabutin without concurrent \\ HIV infection or AIDS.
}

Key words AIDS, HIV, MAC, Rifabutin

Rifabutin-induced uveitis is a recognised complication of opportunistic Mycobacterium avium-intracellulare, kansasii or malmoense therapy in human HIV infection and AIDS. Amongst these individuals, atypical mycobacterial infections occurred in the context of severe immunodepletion and survival was approximately 6-9 months prior to the introduction of highly active antiretroviral combination therapy. Rifabutin-induced uveitis has seldom been described in the absence of HIV infection; however, it has been reported in

MUSHTAQ A. KHAN, JAS SINGH, BALJEAN DHILLON

\section{Case reports}

Case 1

A 46-year-old woman with Mycobacterium avium-intracellulare (MAI) pneumonia was being treated with rifabutin (300 mg o.d.), isoniazid (300 mg o.d.) and ethambutol $(25 \mathrm{mg} / \mathrm{kg}$ ). She presented to the eye department with a 3 day history of a slight ache in her left eye and decreased vision. On examination the patient was systemically well and visual acuity (VA) was $6 / 5$ right eye and hand movements (HM) left eye. The left eye showed a $1 \mathrm{~mm}$ hypopyon with +2 cells and flare in the anterior chamber (AC). Intraocular pressure (IOP) was $14 \mathrm{mmHg}$. The AC activity prevented detailed fundal examination and the disc was just visible with the indirect ophthalmoscope. An AC and vitreous tap was performed but bacteriological, viral and fungal cultures were negative.

Her treatment included cessation of rifabutin and 2 hourly topical steroids with atropine $1 \%$ b.d. to the left eye. The hypopyon resolved and disappeared after 3 days. The resolution of $A C$ activity allowed more detailed fundal examination and a yellowish infiltrate was seen in the vitreous inferonasally in the left eye (Fig. 1). No further intervention was deemed necessary as the VA improved to $6 / 36$ on the above treatment and the patient was observed. Three weeks after initial presentation the posterior segment infiltrate also resolved (Fig. 2) and the VA has returned to $6 / 6$.

\section{Case 2}

immunosuppression. ${ }^{1}$ Atypical mycobacterial infections are becoming increasingly recognised $^{2}$ as a cause of morbidity amongst patients with chronic lung disease.

Consequently, the use of rifabutin is also increasing in the treatment of atypical mycobacteria resistant to other antimicrobial agents.

Three patients who were treated with rifabutin for atypical mycobacterial infection (and no apparent manifestation of HIV infection or AIDS) are presented who developed severe uveitis with vitreous involvement, which responded to topical steroid therapy.
A 46-year-old man was referred to the eye department with a 1 day history of progressive deterioration of vision in his right eye. The patient was being treated with rifabutin $(300 \mathrm{mg}$ o.d.), clarithromycin (500 mg b.d.), ethambutol (25 mg/ $\mathrm{kg}$ ) and ciprofloxacin (750 mg b.d.) for an atypical Mycobacterium lung infection caused by the species $M$. malmoense. Apart from the lung infection the patient was otherwise systemically well. On presentation the VA was $6 / 60$ right eye and 6/6 left eye. IOP was $8 \mathrm{mmHg}$ right eye, $12 \mathrm{mmHg}$ left eye. Slit-lamp examination showed mild conjunctival congestion and a $1 \mathrm{~mm}$ hypopyon with +2 cells 


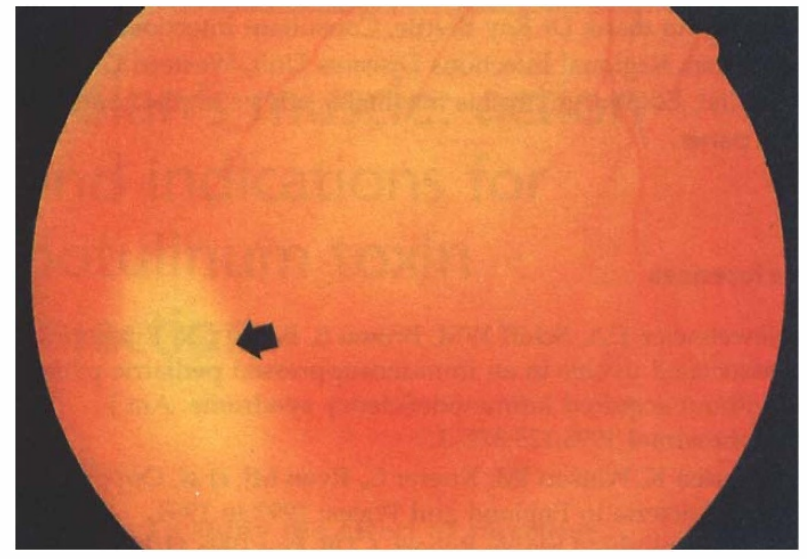

Fig. 1. Case 1. Fundus photograph of the left eye shows a yellowish infiltrate in the vitreous (black arrow).

in the AC. Fundal examination after dilatation of the right pupil was obscured due to the presence of $A C$ inflammation and vitritis. The inflammatory response precluded any fundal photography.

The patient was commenced on 1 hourly g. dexamethasone $0.1 \%$ and g. cyclopentolate $1 \%$ three times a day. One week after the initial presentation there was complete resolution of the hypopyon and the patient's vision improved to $6 / 9$ in the right eye. Fundal examination revealed a discrete inflammatory infiltrate inferiorly in the mid-vitreous. No retinal lesion was seen. Three weeks after treatment was commenced there was complete resolution of inflammation in the AC together with resolution of the discrete inflammatory infiltrate from the vitreous. Vision has improved to $6 / 6$ in the right eye.

\section{Case 3}

A 74-year-old woman was referred to the eye department with gradual deterioration of vision of her left eye over 4 weeks. There was no history of pain. The patient had been on oral steroids for severe chronic obstructive airway disease. She had a past history of MAI infection and her pneumonia was reactivated, requiring treatment with rifabutin ( $300 \mathrm{mg}$ o.d.), clarithromycin $(500 \mathrm{mg}$ b.d.) and ethambutol $(25 \mathrm{mg} / \mathrm{kg})$. On presentation her VA was

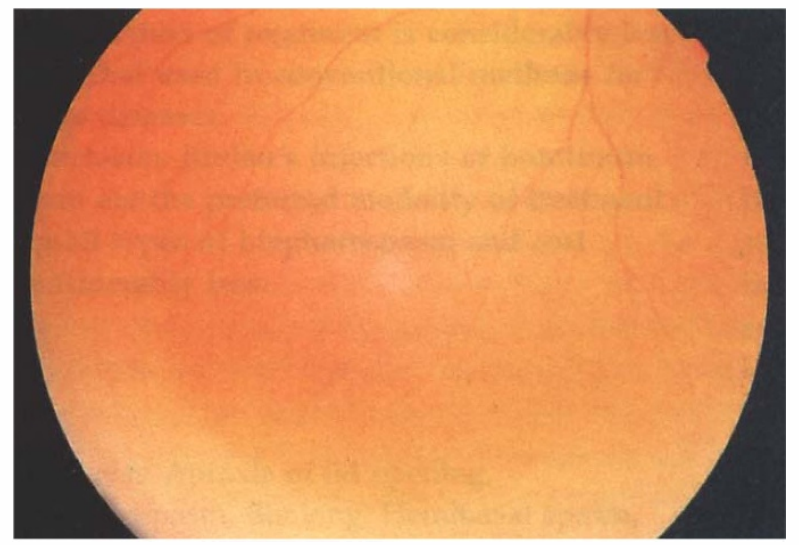

Fig. 2. Case 1. Fundus photograph of the left eye shows complete resolution of the vitreous infiltrate 3 weeks after treatment.

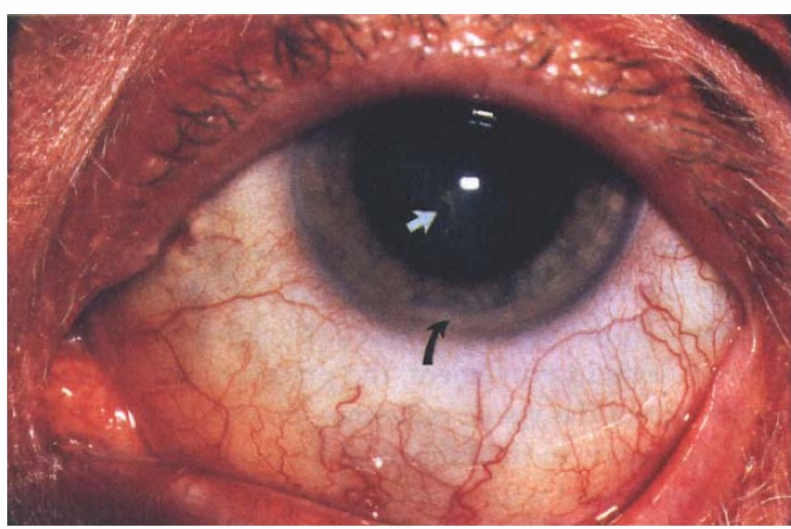

Fig. 3. Case 3. Anterior segment of the left eye shows a hypopyon (black arrow), developing lens opacity and broken posterior iris adhesion (white arrow).

6/18 right eye and HM left eye. Slit-lamp examination of the right eye was normal. The left eye showed no conjunctival congestion but a $1 \mathrm{~mm}$ hypopyon (Fig. 3) with fibrinoid reaction and formation of posterior synechiae. IOP was normal. The patient had a stat dose of phenylephrine $10 \%$ and cyclopentolate $1 \%$ drops, which released the posterior synechiae. On examination of the posterior segment a mid-vitreous yellow-white infiltrate was visible. More detailed fundal examination was not possible due to fibrin clot in the AC and cataract.

The patient was commenced on 1 hourly topical steroids along with twice daily cyclopentolate $1 \%$. The intraocular inflammation responded well to treatment: there was rapid resolution of hypopyon within a week and the vitreous infiltrate resolved after a further week. Four weeks after initial presentation there was complete resolution of ocular inflammation with improvement of VA to $3 / 60$. This poor vision was mainly attributable to her lens opacity. Fundal examination showed complete resolution of infiltrate. Topical treatment was then gradually tailed off.

\section{Discussion}

Rifabutin-associated ocular inflammation in the form of a uveitis is well documented in patients with HIV infections and AIDS. ${ }^{3}$ We describe three cases of uveitis occurring in patients being treated with rifabutin for atypical Mycobacterium infections without HIV infections or AIDS. In all our patients the uveitis affected primarily the anterior segment with spill-over of the inflammation into the posterior segment. All our patients presented with severely reduced vision but minimal conjunctival reaction and little or no pain or photophobia.

It was felt to be inappropriate to test our patients for HIV in view of the lymphocyte counts in the normal range, absence of transmission risk factors for HIV infection and clinical evaluation by senior attending physicians. Atypical Mycobacterium infections have been reported to cause an infective endogenous endophthalmitis ${ }^{4}$ and we pursued ocular investigations in case 1 to detect any ocular pathogens accounting for the clinical findings. All these tests were negative and we 
did not follow a similar line of investigation for cases 2 and 3. All three patients were commenced on topical corticosteroids for the inflammation and mydriatics to prevent iris adhesions to the lens. The uveitis settled in all 3 patients with topical medication only, suggesting that the inflammation primarily affected the anterior segment and the ciliary body. The visual acuity returned to normal in the patients 1 and 2; though patient 3 showed some improvement, the presence of a cataract compromised the visual outcome.

In summary, individuals with atypical Mycobacterium infections treated with rifabutin may present with painless loss of vision in one eye, which may be a drugrelated inflammatory response. The risk of developing uveitis is increased with concurrent administration of clarithromycin therapy. ${ }^{5}$ In one study potential risk factors for developing uveitis were examined. Of the various parameters examined only baseline body weight predicted the development of uveitis. The incidence of uveitis was $14 \%$ in patients weighing $>65 \mathrm{~kg}, 45 \%$ in patients between 55 and $65 \mathrm{~kg}$ and $64 \%$ in patients $<55 \mathrm{~kg}^{6}$

The adverse ocular reaction may be an immune phenomenon caused by dead or dying organisms within the ciliary body releasing immunogenic proteins. This may explain the response to topical therapy alone (and the relative sparing of posterior chorioretinal tissues). Early recognition of this entity can prevent invasive ocular procedures and treatments. Patients tend to recover vision by the use of topical medications alone and may not require the cessation of the inciting drug. ${ }^{7,8}$ We would recommend that any patient receiving rifabutin who has markedly reduced acuity be assessed by an ophthalmologist immediately.
We wish to thank Dr Ray Brettle, Consultant Infectious Disease Physician, Regional Infectious Diseases Unit, Western General Hospital, Edinburgh, for his invaluable advice on the content of this paper.

\section{References}

1. Jewelewicz DA, Schiff WM, Brown S, Barile GR. Rifabutinassociated uveitis in an immunosuppressed pediatric patient without acquired immunodeficiency syndrome. Am J Ophthalmol 1998;125:872-3.

2. Lamden K, Watson JM, Knerer G, Ryan MJ, et al. Opportunist mycobacteria in England and Wales: 1992 to 1994. Communicable Disease Report. CDR Rev 1996;11:147-51.

3. Chaknis MJ, Brooks SE, Mitchell KT, Marcus DM. Inflammatory opacities of the vitreous in rifabutin-associated uveitis. Am J Ophthalmol 1996;122:580-2.

4. Ambler JS, Meisler DM, Zakov ZN, Hall GS, et al. Endogenous Mycobacterium chelonae endophthalmitis. Am J Ophthalmol 1989;108:338-9.

5. Kelleher P, Helbert M, Sweeney J, Anderson J, et al. Uveitis associated with rifabutin and macrolide therapy for Mycobacterium avium intracellulare infection in AIDS patients. Genitourin Med 1996;72:419-21.

6. Shafron SD, Singer J, Zarowny DP, Deschenes J, et al. Determinants of rifabutin-associated uveitis in patients treated with rifabutin, clarithromycin and ethambutol for Mycobacterium avium complex bacteremia: a multivariate analysis. Canadian HIV Trials Network Protocol 010 Study Group. J Infect Dis 1998;177:252-5.

7. Jacobs DS, Piliero PJ, Kuperwaser MG, Smith JA, et al. Acute uveitis associated with rifabutin use in patients with human immunodeficiency virus infection. Am J Ophthalmol 1994;118:716-22.

8. Saran BR, Maguire AM, Nicolas C, Frank I, et al. Hypopyon uveitis in patients with acquired immunodeficiency syndrome treated for systemic Mycobacterium avium complex infection with rifabutin. Arch Ophthalmol 1994;112:1159-65. 